\title{
NETWORK CELL ROUTING MODEL FOR CONTROL OF THROUGHPUT AND DELAY OF AIR TRAFFIC
}

\author{
Alex T. Nguyen and John S. Baras, \\ University of Maryland, College Park, MD
}

\begin{abstract}
Attempts to manage air traffic by either decreasing delay or increasing flow can have the reverse effect on the other. In the National Airspace System (NAS), flights typically depart at their chosen times, and flow management techniques are implemented in the air in an effort to maximize flow, which could lead to delay for individual aircraft. Other areas of the world allow flights to depart only at predetermined slot times knowing that there is a clear unobstructed path, leading to minimal delays but possibly underutilizing airspace.
\end{abstract}

We propose a new approach in leveraging the highway cell transmission model (CTM) to the airspace in the form of a multi-objective optimization that trades between maximizing throughput and minimizing delay. The model is posed as a multicommodity traffic flow integer program where the constraints are relaxed slightly from the CTM to examine strategies for achieving optimal throughput and delay.

In practice, the model is envisioned to initially run offline to determine a preliminary solution to the initial state of the system. Solutions for subsequent changes in actual state can be determined by running the model online for the incremental state change. Stochastic events such as convective weather clearing times or capacities can be included in the model to better see the benefits and impacts of pre-positioning traffic to take advantage of possible future clearing.

\section{Introduction}

In this paper we present a traffic flow model that is inspired by the Cell Transmission Model (CTM) from highway traffic. Lighthill-Whitham-Richards (LWR) initially proposed a set of partial differential equations (PDE) that described the dynamics of highway traffic flow [1] [2]. This was later used by Daganzo [3] [4] to create the Cell Transmission Model, in which a series of consecutive cells is used to represent increments of a highway. The traffic flow from one cell to the next in the simulation model is governed by a discretized approximation of the flow concentration curve from LWR. This model has been widely used in highway transportation for a variety of purposes, such as recent work on networked signal control. [5]

Recently applications of this model to air traffic have included the $\mathrm{CTM}(\mathrm{L})$ model which imposes capacities on a set of cells. [6] The model uses as a decision variable the amount of flights to retain in a cell, and moves other flights to the adjacent cell. The objective function minimizes the usage in the network, and the network consists of fixed predetermined paths between each origin-destination pair.

We propose a model that dynamically routes traffic through congested areas and optimizes for not only minimal delay through the system, but also trades with maximizing throughput. The model is formulated as a multiobjective integer program with similar structure to a multicommodity traffic flow program. The model leverages the framework of the Cell Transmission Model, but with routing capabilities and two objectives.

Air traffic flow models typically establish a node at the intersection of each link, and identify the amount of flow into and out of that node with the assumption that the flow is steady between the nodes. In reality, a bottleneck at a certain point on a link due to weather, heavy traffic, or other reasons can cause backups and increased miles in trail restrictions on aircraft heading to the area.

The dynamics of aircraft movement from cell to cell would be beneficial for tactical actions. This could be used in combination with higher-level flow models that in combination would provide for strategic planning of traffic flows around congested areas. While the specific dynamics of individual vehicles on a highway are not all the same as those for aircraft in the air, we can examine the application of the overall framework of the CTM to the air without modeling individual aircraft. 
Application of the CTM would provide more intuition into the dynamics of the congestion along each segment which would not typically be obvious when using a typical flow model that only includes nodes at intersections. Knowledge of where and when aircraft would be impacted by the congestion downstream, how many would be affected, and their speed reductions or vectors are important in the management of the traffic.

\section{CTM Overview}

In the general CTM model, each aircraft is on an independent path $p$ (treated as a single commodity) from an origin airport to a destination airport.

Links are broken into cells, numbered from 1 to $i$ going in the downstream direction (direction of travel). The length of each cell can be set to be such that without congestion, vehicles would travel from one cell to the next when the time goes from $t$ to $t+1$.

\section{$\leftarrow$ downstream}

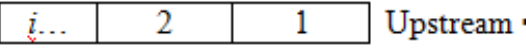

There is only one-way travel on each section of cells. This is acceptable since in the National Airspace System (NAS) the routes are one-way for particular altitude levels.

The state variable is $n_{i}(t)$, the number of vehicles contained in cell $i$ at time $t$. The flow is controlled by $y_{i}(t)$, the number of aircraft that is to flow into cell $i$ in the time interval $(t, t+1)$. The state transition equation can be written simply as

$$
n_{i}(t+1)=n_{i}(t)+y_{i}(t)-y_{i+1}(t)
$$

In the CTM, the relationship between the flow rate and traffic density is derived from a discretized version of the PDEs from the LWR model. [4] The flow rate is approximated by a trapezoidal function of the traffic density. When there is free flow, the total flow is proportional to the number of vehicles in the cell. When the traffic density increases, the flow levels off and is capped at a certain maximal flow amount. As traffic further increases beyond the amount that yields the maximum flow, the flow begins to decrease. Air traffic is not as elastic a highway traffic in the way that the flow cannot reach to zero as traffic levels increase. However, there are small speed controls that can be implemented to slightly lower aircraft speed during areas of high congestion. Alternatively, effective speed reduction or holding within a cell can be viewed as aircraft vectoring to reduce the speed component along the direction of travel.

The inflow $y_{i}(t)$ is thus expressed as

$$
y_{i}(t)=\min \left\{n_{i-1}(t), Q_{i}(t), N_{i}(t)-n_{i}(t)\right\}
$$

where the parameters used are:

$$
\begin{aligned}
& N_{i}(t) \quad \begin{array}{l}
\text { max number of vehicles that can be } \\
\text { present in cell } i \text { at time } t
\end{array} \\
& Q_{i}(t) \quad \begin{array}{l}
\text { max number of vehicles that can flow } \\
\text { into cell } i \text { when the clock advances from } \\
\mathrm{t} \text { to } t+1
\end{array}
\end{aligned}
$$

The two equations above describe the density of flights in the cells and how flights flow from one cell to the next.

\section{Application to Air Traffic Routing}

Here we apply the CTM to an optimization form for use with aircraft in the air. The intent is to model the aircraft flow rather than optimal ground holding strategies. However, results to improve network flow will inherently lead to certain amount of flights being held on the ground in order to optimize delay and throughput in the air.

The flow equation from the CTM has a $\min \{\}$ operator that requires the flow to be no greater than any of the three terms, and also equal to one of the three terms. In order to optimize the delay and throughput, we relax the $\min \{\}$ operator to allow $y_{i}(t)$ to take on values less than all of the terms. In doing so, flights can take a cautious approach and not advance as quickly if there is a possibly of a decrease in capacity downstream. Flights can even remain out of the airborne network and remain on the ground without incurring high costs. This minimizes the airborne delay by not having planes take off and wait in the air at bottleneck points.

To construct the network for the model, the main flows of traffic in the airspace can first be determined via a clustering method. These paths are then converted into a network of cells which can model the movement of aircraft along each route and propagate delays due to weather or other flow 
constrained areas to determine the effects different control methods have on throughput and delay.

The network is set up such that each aircraft is destined for a particular destination airport, and may take different paths to reach the destination to avoid congestion and delays. Figure 1 shows a sample network with two origins and two destinations. A bottleneck exists in cell 3, and causes the majority of traffic from Origin 1 to Destination 1 that would ordinarily travel along cells $2 \rightarrow 3 \rightarrow 5$ to instead travel along the path $2 \rightarrow 8 \rightarrow 4 \rightarrow 5$.

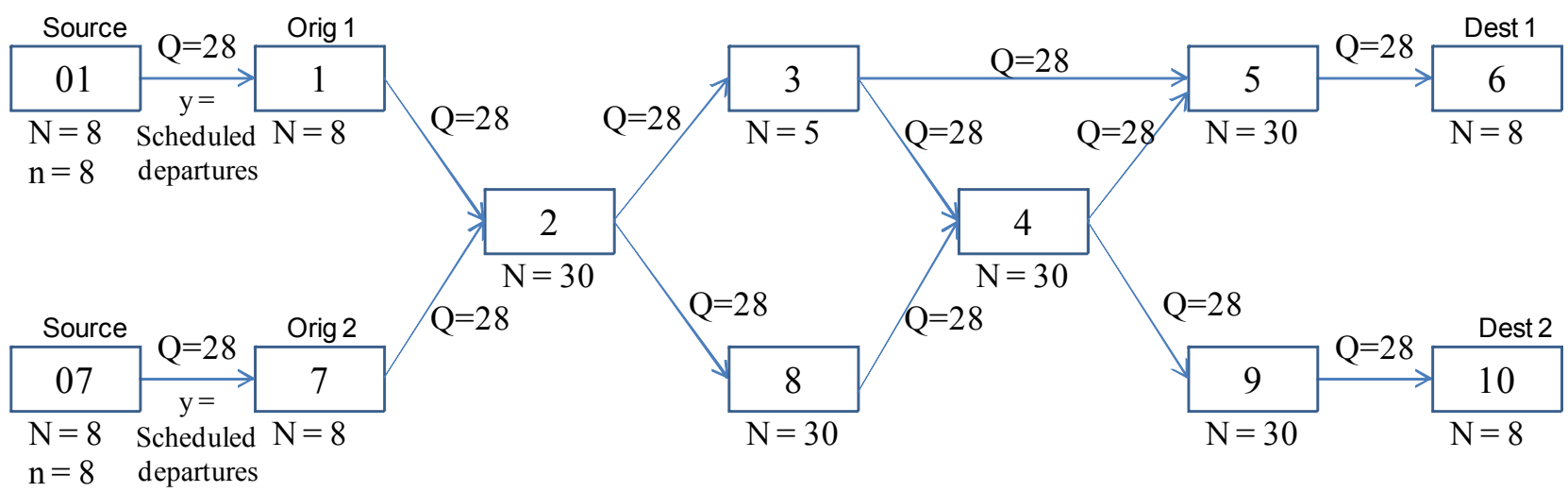

Figure 1. Sample Network Setup

Repository cells are included in front of the origin airports to provide a source of aircraft. As flights are scheduled to depart, they are sent from the repository cells to the departing airport by setting $y_{i, j}^{d}(t)$ to be the number of flights scheduled to depart for destination $d$ during the time period $t$ to $t+1$.

At the origin airport, they could be delayed on the ground before taking off if congestion exists in the network. The origin airport is assumed to have infinite capacity for aircraft to incur delay prior to departure. Similarly, a sink cell can be added after each destination for the flights to move away from the destination once they arrive.

For the problem with multiple commodities and routing, the state and flow variables are redefined as:

$$
\begin{array}{ll}
n_{i}^{d}(t) \quad \begin{array}{l}
\text { number of vehicles contained in cell } i \\
\text { at time } t \text { going to destination } d
\end{array} \\
y_{i, j}^{d}(t) \quad \begin{array}{l}
\text { flow from cell } i \text { to cell } j \text { in the time } \\
\text { interval }(t, t+1) \text { that are destined for } d .
\end{array}
\end{array}
$$

$N_{i}(t)$ max number of vehicles that can be present in cell $i$ at time $t$

$Q_{i}(t)$ max number of vehicles that can flow into cell $i$ when the clock advances from $t$ to $t+1$

$l(d) \quad$ Index of the cell at destination $d$.

$G_{s} \quad$ Set of sinks (the cells that are immediately after the destination cells).

$R_{i} \quad$ Set of cells adjacent to cell $i$ from which flights can flow into cell $i$.

$S_{i} \quad$ Set of cells adjacent to cell $i$ that flights can move to from cell $i$.

$A^{d} \quad$ Total no. of arrivals for destination $d$.

$a_{i, j} \quad$ Indicates whether there is a valid path from cell $i$ to an adjacent cell $j$.

The capacity values for aircraft density and flow are allowed to vary by location and time in order to model changes in weather patterns, special use airspace, or other congestion.

The parameters are: 
We desire to minimize the delay while also maximizing the throughput. A measure of delay can be expressed as the total amount of time the aircraft spend in the system. Throughput can be determined by the number of aircraft that exit the system. Several options exist for optimizing several objective functions. In this model we use the weighting factor $w_{f}$ for the throughput function and combine the two functions into the single objective. The total usage is minimized minus a weighted factor of the total throughput. We could instead maximize throughput minus the usage, but the values for usage tend to be large and could result in negative objective values.

The model is formulated as follows:

$$
\min \sum_{i \neq G s} c_{i} \sum_{d} \sum_{t} n_{i}^{d}(t)-w_{f} \sum_{d} \sum_{t} \sum_{j \in R_{d}} y_{l(d), l(d)+1}^{d}(t)
$$

Subject to:

$$
\begin{array}{ll}
n_{i}^{d}(t+1)=n_{i}^{d}(t)+\sum_{k \in R_{i}} y_{k, i}^{d}(t)-\sum_{j \in S_{i}} y_{i, j}^{d}(t) \\
\sum_{j \in S_{i}} y_{i, j}^{d}(t) \leq n_{i}^{d}(t) & \forall d, i, t \\
\sum_{d} n_{i}^{d}(t) \leq N_{i}(t) & \forall d, i, t \\
\sum_{d} \sum_{j \in R_{i}} y_{j, i}^{d}(t) \leq Q_{i}(t) & \forall i, t \\
n_{i}^{d}(t), y_{i, j}^{d}(t) \in \square &
\end{array}
$$

A cost factor $c_{i}$ can be included in the delay function for each cell if certain routes are desired more than others. In addition, a higher cost can be applied to all cells except the origin cells on the ground to encourage flights remain on the ground to reduce air delay.

The flow conservation equation for each destination and cell is captured in Constraint 1. This shows that the number of aircraft in the next time increment is equal to the number currently in the cell, plus the amount coming in from various cells, minus the amount exiting.

Constraints 2-4 essentially correspond to the terms in the $\min \{\}$ function in the CTM model.
Constraint 2 ensures that the amount of flow leaving a cell cannot be greater than that currently in the cell.

Up to now, each destination, or commodity, is handled independently. Constraint 3 provides a capacity for the flights in a particular cell, across all destinations. An similar capacity constraint (4) can be included to limit the amount of flow into a particular cell for all commodities.

Additional constraints need to be added to ensure that flights only go to their intended destination. This could be done by forcing the total number of arrivals at each destination over all time to be equal to the departures as follows:

$$
\sum_{t} n_{l(d)}^{d}(t)=A^{d} \quad \forall d
$$

This constraint requires that there be enough time for all flights to reach their destination, and would fix the throughput. Alternatively, this could be done by restricting flights from arriving at destinations that are not intended for them.

$$
\sum_{t} \sum_{h \neq d} n_{l(d)}^{h}(t)=0 \quad \forall d
$$

This constraint does not restrict all flights to actually reach the destination in the time allotted, thus allowing for variations in throughput based on the amount of time available in the model. The constraint, though, may cause flights to wander around taking the path of least resistance in any direction if it can't reach the destination within the modeling time, but the minimal usage / delay objective should limit that.

In addition, another constraint needs to be added to force arrivals to move to the adjacent sink at each destination so they don't linger at the destination over time to satisfy arrival constraint.

$$
y_{l(d), l(d)+1}^{d}(t)=n_{l(d)}^{d}(t) \quad \forall d, t
$$

For execution in a solver program, the model may be modified with additional parameters and modifications in the set of indices for the various terms as follows:

$$
\begin{gathered}
n_{i}^{d}(t+1)=n_{i}^{d}(t)+\sum_{k} a_{k, i} y_{k, i}^{d}(t)-\sum_{j \neq i} a_{i, j} y_{i, j}^{d}(t) \\
\forall d, i, t
\end{gathered}
$$




$$
\begin{array}{ll}
\sum_{j \neq i} a_{i, j} y_{i, j}^{d}(t) \leq n_{i}^{d}(t) & \forall d, i, t \\
\sum_{d} n_{i}^{d}(t) \leq N_{i}(t) & \forall i, t \\
\sum_{d} \sum_{j} a_{j, i} y_{j, i}^{d}(t) \leq Q_{i}(t) & \forall i, t
\end{array}
$$

\section{Results \& Discussion}

The model provides a means to tradeoff between reducing delay and maximizing throughput. By adjusting the weighting factor in the objective function, numerous solutions can be obtained with varying degrees of delay and throughput.

Figure 2 shows a sample output showing the resulting delay (represented as usage in the system) and throughput for varying values of the weighting factor $w_{f}$.

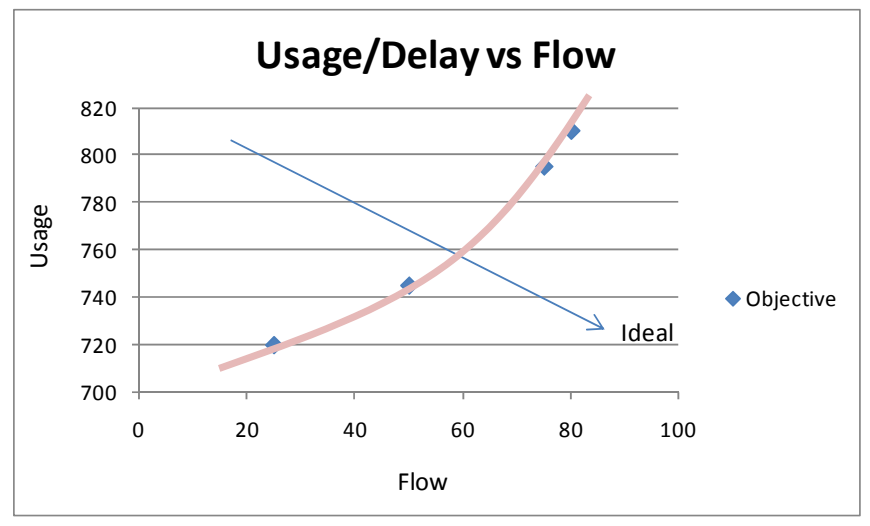

Figure 2. Delay vs Throughput Tradeoff

The usage/delay vs flow curve shows a tradeoff between the two objectives, with the desired direction being towards greater flow and lower usage. The "knee" in the curve indicates a tuning in the model that possibly provides an optimal balance between the two objectives. Decision makes can use this to tailor the model to the desired tradeoff.

This model provides the initial framework for inclusion of additional features such as stochasticity, possibly in the form of parameters. It would be ideal if an integer solution could be obtained in an efficient manner in order to handle additional complexities later. Models that have a constraint matrix that is totally unimodular will have relaxations that yield integer solutions and can be solved quickly.

Typical multicommodity flow models usually do not have constraint matrices that are totally unimodular, with the exception of those that have either two or less sources or sinks [7]. The constraint matrix for this problem has the following form:

$$
\mathbf{A}=\left[\begin{array}{cc}
\mathbf{N}_{1} & \Upsilon_{1} \\
\mathbf{N}_{2} & \mathbf{0} \\
\mathbf{0} & \Upsilon_{2}
\end{array}\right]
$$

All elements $a_{i j}$ in $\mathbf{A}$ have $a_{i j} \in\{-1,0,+1\} . \mathbf{N}_{1}$ and $\Upsilon_{1}$ have columns that either have all 0 's or have exactly one +1 and one -1 . With just these two submatrices, the constraint matrix would be totally unimodular. However, $\mathbf{N}_{2}$ and $\Upsilon_{2}$ are also included, and correspond to the flow capacity constraints across the multiple destinations. While a single identity submatrix below the flow constraints would preserve the total unimodularity of $\mathbf{A}$, these two submatrices have the form $\left[\begin{array}{llll}\mathbf{I} & \mathbf{I} & \mathbf{I} & \ldots\end{array}\right]$. However, not satisfying the usual sufficient condition for total unimodularity does not mean that the problem cannot yield integer solutions. It is possible that in some cases the linear program relaxation may produce integer solutions. This would be an ideal situation for computational efficiency. This model, while similar to a multicommodity flow model, has more structure that may lead it to yield integer solutions from the relaxation. The solutions derived thus far on a small number of samples used seem to indicate some optimism for integer solutions for the model.

The actual computational efficiency for a small sample is shown in Figure 3. The increase in computation time relative to the network size is shown along with the respective number of airports used in the scenario. The computation time appear to yield well with the network size and airport size. However, this may vary greatly depending on the specific network structure. 


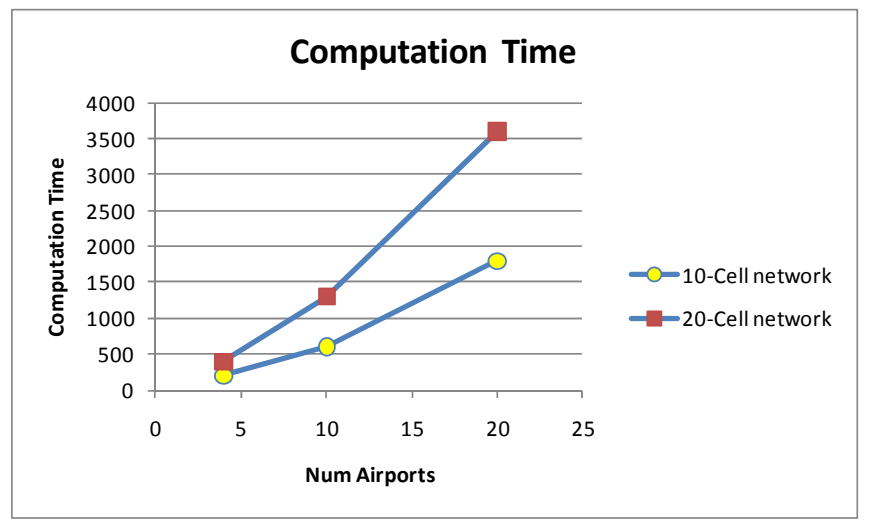

Figure 3. Computation Time

\section{Conclusions \& Future Work}

A model has been developed that models the movement of flights across a network and provides for a tradeoff between minimal delay and maximal throughput. Inspired by the Cell Transmission Model, this model captures the movement of aircraft from cell to cell within a network, and determines the optimal routing of aircraft through congested areas.

Ongoing work on the model include determining efficient integer solutions to the problem, as well as making the model stochastic with inclusion of parameters to model randomness in flight departure times and its effect on delay and throughput.

\section{References}

[1] Lighthill, M.J., J.B. Whitham, 1955, "On Kinematic Waves. I Flow movement in long rivers.
II A theory of Traffic Flow on Long Crowded Roads", Proc. Royal Society, A 229, pp. 281-345.

[2] Richards, P.I., 1956, "Shockwaves on the Highway", Operations Research, Vol. 4, No. 1, pp. 42-51.

[3] Daganzo, C., 1994. The cell transmission model: A dynamic representation of highway traffic consistent with the hydrodynamic theory," Transportation Research Part B, Vol. 28B, No.4, pp. 269-287.

[4] Daganzo, C., 1995. "The cell transmission model, Part II: Network traffic", Transportation Research Part B, 29(2).

[5] Gabriel G., Roberto Horowitz, Alex Kurzhanskiya, Pravin Varaiya, Jaimyoung Kwon, 2008, "Behavior of the cell transmission model and effectiveness of ramp metering", Transportation Research Part C 16, 485-513.

[6] Sun, D., Alexandre Bayen, "Multicommodity Eulerian-Lagrangian Large-capacity Cell Transmission Model for en route traffic," AIAA Journal of Guidance, Control and Dynamics, vol. 31, no. 3, pp. 616-628, 2008.

[7] Evans, J. R., J. J. Jarvis and R. A. Duke, "Matroids, Unimodularity, and the Multicommodity Transportation Problem," Presented at the Joint ORSA/TIMS Meeting, Chicago, 1975.

28th Digital Avionics Systems Conference

October 25-29, 2009 